\title{
Competencia digital: una necesidad del profesorado universitario en el siglo XXI
}

\section{Digital competence: a need for university teachers in the 21 st century}

\author{
$\mathrm{M}^{\mathrm{a}}$ Paz Prendes Espinosa \\ Universidad de Murcia. Murcia (España) \\ pazprend@um.es \\ Isabel Gutiérrez Porlán \\ Universidad de Murcia. Murcia (España) \\ isabelgp@um.es \\ Francisco Martínez Sánchez \\ Universidad de Murcia. Murcia (España) \\ pacomar@um.es
}

\begin{abstract}
Resumen
Este artículo presenta un análisis de la situación actual de las universidades en España y su dotación tecnológica para, a partir del mismo, poder analizar la situación del profesorado universitario. Partiendo del concepto de competencia profesional, analizamos la competencia profesional del docente para posteriormente adentrarnos en la competencia digital docente. Tras realizar una revisión sistemática de los modelos de competencia digital docente universitaria, realizamos una propuesta original que pretende aunar todas las dimensiones que a lo largo de estos años han ido proponiendo los diversos autores que han afrontado la tarea de definir sus propios modelos. Nuestro modelo se sustenta en cinco dimensiones (técnica, informacional/comunicativa, educativa, analítica y socio-ética) así como en tres ámbitos de aplicación (docencia, investigación y gestión). Esta propuesta innovadora puede ser objeto de reflexión y análisis, pero también puede sustentar investigaciones empíricas en un futuro próximo.
\end{abstract}

\section{Palabras clave}

Competencia digital docente, profesor universitario, modelo de competencia digital.

\begin{abstract}
This article presents an analysis of the current situation of Spanish universities and their technological infrastructure. This analysis is the first step to reflect on the situation of university teachers. We focus on teachers' professional competence in order to revise their teaching competence, which will allow us to examine different models of teaching digital competence at university. This systematic review is useful when presenting our own proposal. After collecting data on all the dimensions described in previous models, we build a new model based on five dimensions (technical, information and communication, educational, self-reflection, and social and ethical) and three fields of application (teaching, research, and management). This new model may be a useful tool for future empirical research while it may promote critical reflection.
\end{abstract}

Key words

Digital competence, university teacher, digital competence model. 


\section{Las universidades en España}

Uno de los grandes cambios que encontramos en la sociedad actual ha sido el desarrollo de los medios de comunicación, lo que ha supuesto un proceso de globalización, que con sus luces y sus sombras repercute en todo lo que los humanos hacemos en estos momentos. Desde la economía a la cultura, desde el conocimiento al ocio, desde el deporte a la gastronomía, la globalización va creando nuevas situaciones de relación humana que, consciente y/o inconscientemente, vamos asumiendo en nuestro quehacer cotidiano. Estos cambios deben repercutir inevitablemente en la Universidad, que sin dejar de ser lo que fue, ha de adaptarse a la nueva situación, y aquí es donde puede radicar una de sus debilidades o cuando menos su disfunción en algunos momentos.

La Universidad ha seguido aferrando a la especialización, por supuesto necesaria en la investigación, pero posiblemente no lo sea tanto en cuanto a la formación y la docencia. Todorov (2008, p. 55) nos decía que: "El saber es indispensable, pero es un medio", un medio necesario, pero evidentemente no exclusivo ni puede convertirse en el único fin de la acción docente en las universidades.

Como puede verse la preocupación por la transformación de las enseñanzas y con ellas de la formación no es una preocupación actual, viene de tiempo atrás, si bien durante todo ese tiempo no se han realizado muchos de los cambios que habría sido necesario abordar para adecuar las universidades a la nueva situación, tanto desde la perspectiva de la investigación como, y en este caso que nos ocupa, la docencia y con ella los objetivos de la formación que se imparte, el qué, el para qué y el cómo de la acción docente universitaria.

Por otro lado, la estructura organizativa de estas instituciones, los procesos para la toma de decisiones, los intrincados caminos para la modificación de los planes de estudios y con ellos sus contenidos, los procedimientos para la distribución interna de los créditos, etc., muestra una institución lenta de reflejos, mirándose más a ella misma y a sus intereses internos que a la evolución de la sociedad a la que debe atender en sus necesidades. No debemos olvidar que es en las organizaciones, a sus diferentes niveles, desde donde se hace posible o se impide el cambio.

Flexibilidad, agilidad en la toma de decisiones, primar lo general sobre lo particular, mirar con frecuencia y escuchar al "exterior", eliminación de estructuras organizativas cerradas, facilitar la transversalidad de los curricula que se oferten, etc. son algunos de los aspectos en los que la organización ha de pensar y revisar si se pretende adecuar la institución universitaria a la realidad mediatice actual.

\section{Tecnologías en el sistema universitario}

Como una posibilidad de solucionar algunos de los problemas existentes en la docencia universitaria se mira a las Tecnologías de la Información y la Comunicación. Las universidades disponen de tecnologías. Según los datos de la red Eurydice (2011) a partir de un estudio con 35 países, la mayor parte de los centros de enseñanza en Europa cuentan con tecnologías y no hay grandes diferencias en cuanto a 
equipamiento. En este informe se argumenta la importancia de la innovación en las instituciones de enseñanza y, de forma más específica, las innovaciones orientadas a la mejora de la enseñanza y el aprendizaje mediante la utilización de nuevas tecnologías. Situando nuestro punto de mira específicamente en las universidades españolas, los datos de Gómez (2016) vienen a describir esta misma realidad, el equipamiento y las infraestructuras son una condición necesaria pero no son un problema. Una vez que los datos muestran que contamos con la infraestructura tecnológica necesaria para promover cambios y mejoras, será importante concretar hacia dónde queremos que las tecnologías nos lleven.

Por tanto, dotar de sistemas tecnológicos de comunicación avanzados a las aulas es relativamente sencillo y muy vendible socialmente, pero el que estas tecnologías se utilicen adecuadamente y sirvan para algo realmente útil es otra cuestión muy diferente. Aunque también puede haber quienes piensen que, tal como decía Bacon en su "Opus Maius", que la tecnología es el medio de recuperar la perfección original perdida del ser humano y estemos resucitando y/o asignado un "valor trascendental tecnológico".

Las tecnologías forman parte de la realidad que nos ha tocado vivir y en opinión de Marcovitch (2002, p. 87):

"Ante la revolución tecnológica, la universidad se comporta como cualquier otra organización de nuestro tiempo. No puede ignorarla y dejar de aprovechar todos sus beneficios. Evidentemente, como centro crítico y cuestionador por naturaleza, la universidad jamás será una usuaria incondicional de las oportunidades cradas por la tecnología, pero desconocerla o dejar de aprovecharla, cuando se hace necesario, es absolutamente imperdonable".

Por su parte la Comisión Europea (2014) señala que la innovación en cuanto a tecnologías digitales en Europa avanzará en los próximos años en el desarrollo de la robótica y de los componentes y sistemas (electrónica, computación, inteligencia artificial,...). Mientras por su parte, el informe Horizon 2017 (NMC, 2017) hace hincapié en los desarrollos del conocido como Internet de las Cosas. Y éste es el mundo en el cual nos ha tocado vivir y en el cual las universidades han de cumplir con su misión:

\begin{abstract}
"Concretamente, desde distintos organismos internacionales se afirma la necesidad de que la universidad del siglo XXI cree las condiciones adecuadas para fomentar un aprendizaje más centrado en el estudiante y haga uso de métodos de enseñanza innovadores. [...] la nueva coyuntura universitaria europea reclama una cultura pedagógica que no se limite a la mera transmisión de contenidos y a la posterior superación de una prueba evaluativa puntual, sino que forme a los estudiantes como ciudadanos críticos y activos dispuestos a poner su conocimiento al servicio de la sociedad" (Santos Rego et al., 2017, p. 42).
\end{abstract}

Las universidades tienen una responsabilidad con la sociedad, especialmente las universidades públicas por estar financiadas mayormente con fondos públicos. Y en palabras de Marcovitch (2002, p. 22), "la universidad debe formar líderes, agentes de cambio: hombres y mujeres dispuestos a asumir riesgos para construir un mundo mejor". Ningún universitario pondría en duda que la labor del profesorado es un elemento esencial, por lo que vamos a situarlo en el punto central de nuestro análisis. 


\section{Uso de tecnologías para la formación: el profesorado como clave del proceso de innovación en la universidad}

Si más arriba decíamos que el saber es un medio, la tecnología lo es mucho más. Cualquier medio, sea este el que sea, ha de insertarse dentro de un modelo de enseñanza, dentro de una metodología que es la que en último extremo da sentido y justifica los medios que se incorporan. En palabras de Ricoy, Sevillano y Feliz (2008, p. 504), "el escenario del EEES presenta una elevada exigencia para abordar la formación enfocada al desarrollo de competencias tecnológicas y, además, las TIC están llamadas a protagonizar un papel primordial como medios de aprendizaje y recursos de enseñanza". Y para ello, sin duda, uno de los elementos básicos ha de ser el papel del profesorado como agente de cambio y como profesional cuyos procesos de formación a lo largo de la vida estarán inevitablemente ligados al desarrollo de las Tecnologías de la Información y la Comunicación. Esta unión nos conduce de forma directa a analizar la importancia de la competencia digital entre el conjunto de las competencias profesionales de los docentes.

El Informe Horizon 2017 sobre Enseñanza Superior (NMC, 2017) remarca la idea de que la competencia digital no es solamente entender cómo usar las tecnologías, sino que indefectiblemente pasa por la necesidad de comprender el profundo impacto de las tecnologías en un mundo digital y promover la colaboración para integrarlas de modo efectivo. Además insiste de nuevo en la tendencia observada en años anteriores y es la progresiva implantación de los distintos modelos de enseñanza que flexibilizarán nuestro sistema de enseñanza superior (blended-learning, e-learning, m-learning, adaptative learning,...). Por otra parte, destacan la importancia de la formación permanente como modelo que ha de sustentar la capacitación del profesorado y el enfoque de la formación del alumnado. Es la formación permanente y a lo largo de la vida una de las apuestas de este grupo de expertos para el futuro de las instituciones universitarias. Este informe es elaborado anualmente por expertos usando un método Delphi de carácter cíclico (véase la Figura 1).

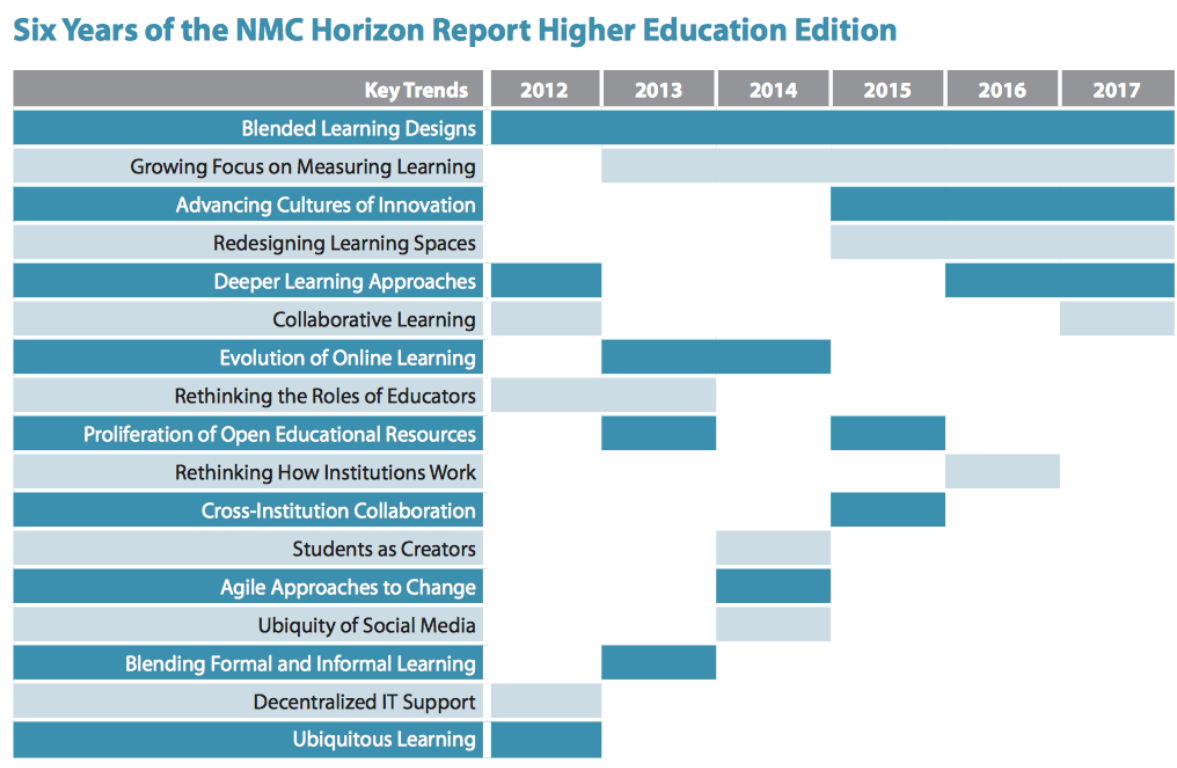


Figura 1: Aspectos clave de la innovación apoyada en TIC en la Enseñanza Superior 2012-2017 (Informe Horizon, NMC, 2017, p. 4)

Es por tanto el docente -que desarrolla una metodología en los procesos de formaciónquien decide el papel que han de cumplir las tecnologías. Lo dicho significa que es el docente quien ha de diagnosticar situaciones de enseñanza, decidir el tratamiento y aplicarlo, lo que conlleva una formación y dedicación acorde con sus responsabilidades. Todo lo dicho significa que el profesor universitario debe disponer de una serie de capacidades acordes con las necesidades actuales y que Aramburuzabala Higuera, Martínez-Garrido y García-Peinado (2013, pp. 17-18) concretan en las siguientes (Figura 2):

- Importancia de las redes de docencia e investigación nacionales e internacionales

- Tendencia hacia el «trabajo colaborativo» y en equipos docentes

- Interés por las cuestiones relacionadas con el tratamiento de la diversidad y la inclusión en la Universidad

- Sensibilidad hacia las temáticas de igualdad y no discriminación en la enseñanza

- Valoración de las competencias relativas al «liderazgo educativo»

- Mayor compromiso e implicación del profesor con los estudiantes

- Desarrollo y valoración del «espíritu emprendedor»

- Mayor estructuración de los procesos de aprendizaje

- Mejora en los procesos de evaluación del aprendizaje

- Evaluación multinivel de todos los procesos de enseñanza

- Generación de una «práctica reflexiva» en los equipos de profesores

- Formalización y reorganización de la carrera docente

- Reforma constante de los sistemas de enseñanza y aprendizaje

- Presencia generalizada de las TIC en la educación

- Necesidad de contar con nuevos dispositivos de formación inicial y permanente del profesorado

Figura 2. Capacitación del profesorado, según Aramburuzabala Higuera, MartínezGarrido y García-Peinado (2013, pp. 17-18).

Coincidimos con Amador y cols. (2017, pp. 13-14) al señalar que en el contexto actual de formación, el profesorado universitario se encuentra con el gran desafío de afrontar su cambio de rol, asumiendo que, además de la función docente vinculada al contenido, ha de desarrollar sus competencias para "guiar, asesorar y crear espacios y oportunidades para que el alumnado pueda desarrollar las competencias profesionales", estando así "inmerso en un proceso de reflexión y análisis de sus propias prácticas docentes". Los autores señalan como referentes claves de la nueva Universidad del siglo XXI las nuevas tecnologías, la interdisciplinariedad y la innovación docente. Los docentes de esta Universidad actual "se convierten en mediadores, diseñadores de entornos de aprendizaje y propiciadores del aprendizaje autónomo de los alumnos, para lo que se le exigen competencias pedagógicas, manejo de técnicas y recursos educativos" (Santos et al., 2017, p. 42).

¿Dados los cambios que se están produciendo en la sociedad y con ello las nuevas exigencias a la formación de los profesionales, está el profesor universitario formado para poder asumir las necesitas actuales de unos objetivos, alumnos y métodos 
diferentes a los convencionales? ¿Se han adquirido, aunque sea por aproximación, las capacidades que se han relacionado en la Figura 1?

Centrándonos en las TIC, podemos ver que en la relación de capacidades apuntada más arriba, buena parte de ellas se apoyan en la disponibilidad de tecnologías de la comunicación que hacen posible su desarrollo, pero centrándonos en estas tecnologías la pregunta que el docente ha de ser capaz de responder es clásica pero no por ello se es capaz de responder por todos los docentes: ¿por qué este medio aquí y ahora?

En una investigación realizada en diversas universidades españolas sobre la formación del profesorado en relación con el uso de tecnologías de la información y la comunicación (Marín, Román, Barroso y Castaño, 2002), se reconoce como una constante la consideración de las tecnologías como recursos de gran relevancia en el contexto de la Universidad, tanto para la formación como para la investigación y la gestión. Y en línea con los argumentos que venimos presentando, los autores destacan que la introducción de TIC es una gran preocupación de los responsables y los gestores universitarios, llegando incluso algunos de ellos a identificar el concepto de innovación educativa con el concepto de innovación con TIC. Una tercera variable a destacar, según este trabajo, es la importancia de los factores organizativos.

En la situación actual del sistema universitario español, es importante también reconocer no solamente la responsabilidad del profesorado, sino la cada vez mayor presión del sistema de evaluación y acreditaciones que no creemos redunde en la calidad del sistema. Es lo que Trillo describe como "los estragos de un perverso sistema de incentivos" (2008, p. 22) en un artículo de opinión que no puede ser más claro y más rotundo. En este trabajo describe la realidad actual de la Universidad española y, tras dejar constancia de las distintas leyes que han ido empeorando cada vez más las instituciones universitarias, analiza la situación del profesorado, la cual describe explicando que "cada vez más nos pasamos el día informando sobre nuestras respectivas actividades y cubriendo para ello numerosos impresos de control para múltiples servicios y agencias, pues ahora todo el mundo vigila a todo el mundo", por lo que "trabajamos horas y horas para una burocracia de administrativos anónimos" (p. 28).

Desde esta perspectiva, se antoja a menudo complicado implantar una auténtica cultura y filosofía de innovación, pues exige un esfuerzo que no siempre estamos dispuestos a hacer. Y todos los cambios en los modelos de formación han de venir aparejados de un planteamiento global de las instituciones, pues tan importantes son los agentes educativos como las instituciones en sus aspectos organizativos, normativos y de liderazgo. En palabras de Salinas (2004, p. 2), "Las universidades necesitan implicarse en procesos de mejora de la calidad y esto, en nuestro terreno, se traduce en procesos de innovación docente apoyada en las TIC". Las universidades han de reflexionar seriamente sobre su situación actual y sobre cuál ha de ser el camino de la innovación y el cambio que quieren abordar desde sus planes estratégicos y sus objetivos estructurales para corto, medio y largo plazo. Además "cada Universidad ha de responder desde su propia especificidad, partiendo del contexto en que se halla, considerando la sociedad a la que debe servir, teniendo en cuenta la tradición y las fortalezas que posee" (Salinas, 2004, p. 2). 
Es igualmente relevante reseñar, tal y como enfatiza el informe UNESCO (2004), que el éxito de la innovación con TIC en la formación universitaria exige la implicación tanto de profesorado como de alumnado, pues ambos agentes deben tener acceso a las tecnologías, deben disponer de contenidos educativos digitales de calidad que respondan a la diversidad cultural y por último, deben poseer habilidades y conocimientos para el uso de tecnologías.

Son los usuarios quienes desde sus propias prácticas innovadoras acabarán exigiendo e "imponiendo cambios tecnológicos a las instituciones" (Cebrián, 2013, p. 504) a la par que las instituciones pueden liderar e incentivar las políticas de innovación. Políticas de innovación que en los últimos años han venido íntimamente unidas a las TIC y en el ámbito de la formación, han venido unidas a la implantación de modalidades de enseñanza flexible que nos conducen hacia la necesaria introducción de TIC en la Universidad.

\section{La competencia profesional del docente universitario}

Hacer referencia a competencias profesionales nos conduce a poner en el punto de mira la relevancia del contexto donde uno ha de ejercer su labor profesional y donde ha de demostrar que realmente tiene la competencia. En palabras de Tejada y Navío (2005, p. 5), "la utilidad de la competencia profesional está en la capacidad de ésta para hacer frente a contextos profesionales cambiantes y en los que aspectos como la polivalencia y la flexibilidad son necesarios" y se puede adquirir y desarrollar gracias a la formación inicial, a la formación continua y a la propia actividad profesional. Partiendo del trabajo de Zabalza (2003), podemos afirmar que la competencia profesional hace alusión a un constructo molar referido a un conjunto de conocimientos y habilidades para que las personas puedan desarrollar algún tipo de actividad.

No vamos a hacer un análisis exhaustivo del concepto de competencia profesional del docente, pues excede el objetivo de este artículo, pero sí nos parece interesante recoger la definición de Escudero (2006. p. 34), quien entiende que la competencia docente es:

"conjunto de valores, creencias y compromisos, conocimientos, capacidades y actitudes que los docentes, tanto a título personal como colectivo (formando parte de grupos de trabajo e instituciones educativas) habrían de adquirir y en las que crecer para aportar su cuota de responsabilidad para garantizar una buena educación a todos"

El mismo autor analiza las competencias docentes y elabora una propuesta que incluye tres categorías de la competencia: el conocimiento de base (sobre aprendizaje, diversidad, contenidos y metodologías); las capacidades para enseñar (planificar, diseñar, comunicar, enseñar, evaluar); y la responsabilidad profesional (ética, responsabilidad, reflexión, liderazgo y colaboración).

Por su parte Zabalza (2007) considera que la competencia docente ha de permitir a un profesor planificar la enseñanza, seleccionar y organizar los contenidos, presentar la información, seleccionar metodologías, diseñar tareas, interactuar con el alumnado, realizar la labor tutorial, investigar, reflexionar y sentirse miembro de la institución. Este modelo es utilizado por Flores y Roig (2016) como base para analizar la 
competencia digital docente, entendiendo que ésta permitirá al profesorado ejercer su labor profesional utilizando las TIC para llevar a cabo todas esas acciones en las que resulta necesaria la competencia profesional. Abundaremos en este concepto de competencia digital docente más adelante.

Hemos comparado el análisis y las clasificaciones de la competencia docente de diversos autores (Salinas, 1998; Zabalza, 2003 y 2007; Perrenoud, 2004; Escudero, 2006; Tejada, 2009; Gutiérrez, 2011; Mas, 2011; Pagés y cols., 2016) y como síntesis de todas estas propuestas resulta la Tabla 1.

Tabla 1: La competencia profesional del docente

\begin{tabular}{|c|c|}
\hline Competencia disciplinar & $\begin{array}{l}\text { Conocimiento de la disciplina } \\
\text { Actualización del conocimiento } \\
\text { Lenguaje propio de la disciplina }\end{array}$ \\
\hline $\begin{array}{l}\text { Competencias para gestionar } \\
\text { la enseñanza }\end{array}$ & $\begin{array}{l}\text { Planificación de la enseñanza } \\
\text { Diseño de tareas y organización de actividades } \\
\text { Selección de contenidos y elaboración de recursos } \\
\text { Habilidades comunicativas (explicaciones e interacción didáctica) } \\
\text { Uso de metodologías diversas y modelos docentes centrados en el } \\
\text { alumno } \\
\text { Control y regulación de la docencia, autoevaluación }\end{array}$ \\
\hline $\begin{array}{l}\text { Competencias para gestionar } \\
\text { el aprendizaje }\end{array}$ & $\begin{array}{l}\text { Implicar al alumnado, fomentar la participación y acceso a la } \\
\text { información } \\
\text { Interacción con el alumnado } \\
\text { Tutoría y orientación del alumno } \\
\text { Conocimiento del proceso de aprendizaje } \\
\text { Control y regulación de los aprendizajes } \\
\text { Evaluación y feed-back }\end{array}$ \\
\hline Competencias tecnológicas & $\begin{array}{l}\text { Utilización de recursos y nuevas tecnologías (en docencia, } \\
\text { investigación y gestión) } \\
\text { Integración de tecnologías en situaciones de enseñanza diversas } \\
\text { Gestionar ambientes de aprendizaje flexibles y enriquecidos con } \\
\text { tecnologías } \\
\text { Innovación apoyada en tecnologías }\end{array}$ \\
\hline $\begin{array}{l}\text { Competencias para la } \\
\text { colaboración }\end{array}$ & $\begin{array}{l}\text { Trabajo en equipo } \\
\text { Relaciones interpersonales } \\
\text { Comunicación con agentes educativos y sociales } \\
\text { Colaboración con otros docentes, acceder al conocimiento de } \\
\text { otros expertos }\end{array}$ \\
\hline $\begin{array}{l}\text { Competencias organizativas e } \\
\text { institucionales }\end{array}$ & $\begin{array}{l}\text { Contribuir a la mejora de la formación y a la innovación } \\
\text { Liderazgo } \\
\text { Gestión de organizaciones } \\
\text { Conocimiento de la institución } \\
\text { Identificación con la institución }\end{array}$ \\
\hline $\begin{array}{l}\text { Competencias de desarrollo } \\
\text { profesional }\end{array}$ & $\begin{array}{l}\text { Gestionar el desarrollo profesional y la formación permanente } \\
\text { Autocrítica, reflexión sobre la propia práctica } \\
\text { Ética en el desarrollo profesional } \\
\text { Esperanza, esfuerzo y pasión por la profesión } \\
\text { Investigación, innovación, creatividad }\end{array}$ \\
\hline
\end{tabular}

Quizás de todos los trabajos sobre competencia docente, el más conocido y que mayor impacto ha tenido haya sido el de Mishra y Koehler (2006) con su modelo TPACK (Technological Pedagogical Content Knowledge) sobre el conocimiento tecnológico, 
pedagógico y disciplinar. Mientras el conocimiento disciplinar se refiere a la materia, el conocimiento pedagógico se refiere a la capacidad de gestionar los procesos de enseñanza-aprendizaje y el conocimiento tecnológico hace alusión a la capacidad de usar medios, así como la capacidad para comprender sus posibilidades y limitaciones. Las interacciones de estos tres tipos de competencia acaban configurando, en el círculo central, el modelo ideal de docente competente en los tres ámbitos. Un instrumento de evaluación de la competencia según el modelo TPACK puede encontrarse en Cabero y Barroso (2016) y en Cabero, Marín y Castaño (2015).

En definitiva, en las universidades del siglo XXI, el docente ha de dejar de ser el controlador del acceso a la información del alumno y pasar a ser el alumno el último responsable de ello. Pero sin embargo, "los diferentes instrumentos de evaluación del profesor se centran fundamentalmente en el comportamiento docente y en las variables que pueden afectar su conducta (como el interés de los estudiantes, la valoración de la asignatura, etc.)", en palabras de Villa Sánchez (2008, p. 190), lo que significa que el resultado de estas evaluaciones no puede ser significativo ni un referente para poder decidir sobre las necesidades formativas que puedan significar cambios con relación a las demandas actuales. En buena medida los resultados de estas evaluaciones refuerzan los modelos pedagógicos tradicionales de la Universidad. Continuando con lo que dice Villa Sánchez (2008, p. 191):

"no es lo mismo explicar bien una lección o tema y hacerlo accesible a los estudiantes que planificar una estrategia para que los estudiantes aprendan por sí mismos, orientar su aprendizaje, ofrecerles apoyo y retroalimentación de su propio proceso, evaluar las competencias que adquieren o desarrollan, entre otras tareas."

Y es esta capacidad la que hay que evaluar y sería lo que permitiría conocer realmente las necesidades formativas reales y las carencias formativas reales, con el fin de lograr una transformación docente acorde con las posibilidades que los nuevos sistemas de comunicación ponen a disposición de la enseñanza, así como poder aproximarse al logro de alumnos con las competencias que se requieren en el momento actual.

\section{La competencia digital docente en las universidades}

Los profesores universitarios reconocen su necesidad en cuanto a capacitación tecnológica, como hemos mencionado. Y alcanzar lo que se ha dado en llamar la competencia digital podría ser el objetivo a lograr para poder aproximar al docente universitario a integrar de forma coherente las TIC en su función docente. Las características singulares de la enseñanza universitaria, tanto desde el punto de vista del alumnado como de sus contenidos, su organización y especialización, hace que parezca necesaria una adaptación de los constructos en torno a la competencia docente y la competencia digital que contemplen esas singularidades.

Docentes y discentes en la Universidad no pueden escapar de la realidad y el tiempo en el que vivimos, absolutamente condicionados por las tecnologías y teniendo además presente que la competencia digital es una de las competencias clave del ciudadano del siglo XXI. Estas competencias aparecen representadas en la Figura 3. 


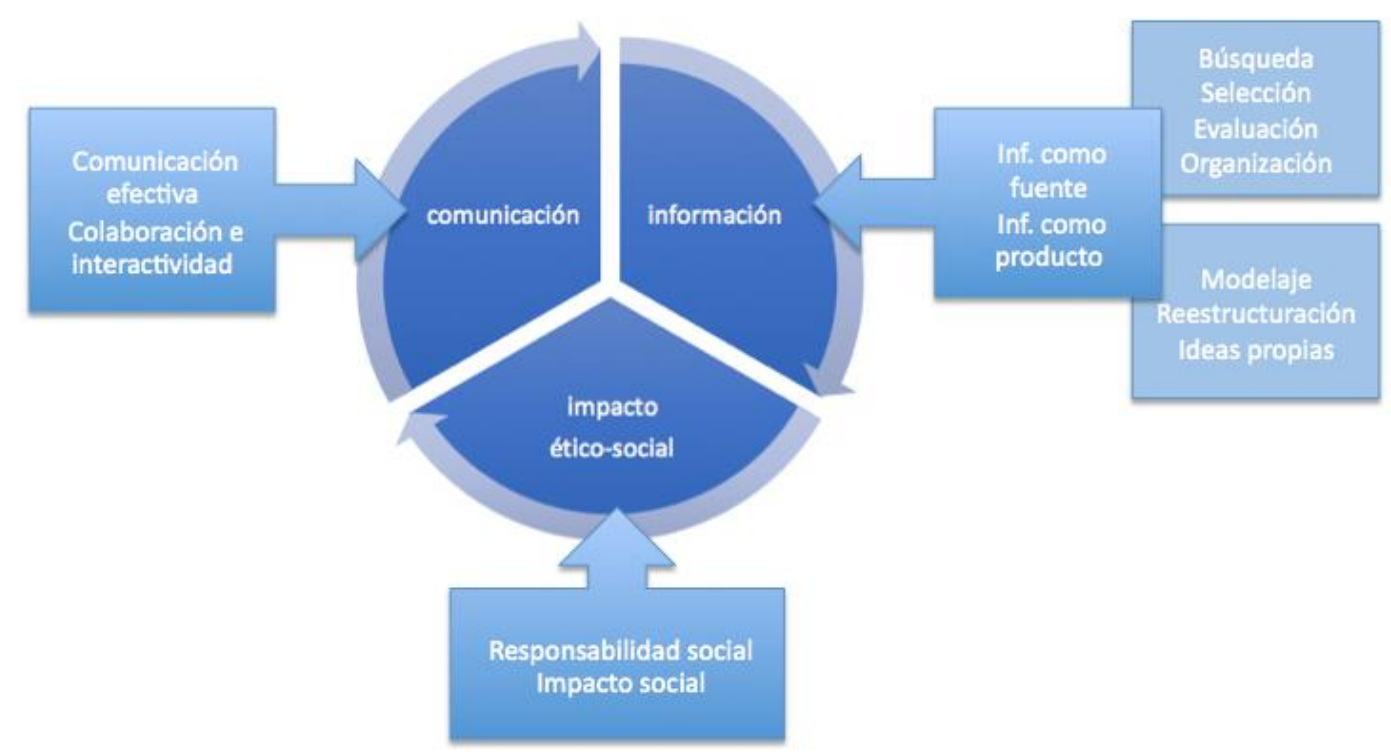

Figura 3: Competencias del ciudadano del siglo XXI, a partir de OCDE (2005) e ITE (2010)

La Comisión Europea $(2007,2016)$ concreta las siete competencias clave del ciudadano del siglo XXI en las siguientes: comunicación en lengua materna y en alguna lengua extranjera; competencia matemática, científica y tecnológica; competencia digital; aprender a aprender, resolución de problemas y creatividad; competencia cívica y social; iniciativa, innovación y emprendimiento; conciencia cultural, inclusión social y pensamiento crítico. Más reciente es la propuesta de UNESCO (2016) sobre la "educación para la ciudadanía mundial" en la cual juega un papel fundamental "el uso de las tecnologías de la información y de los medios de comunicación social para unir a localidades y estudiantes, a docentes y técnicas" (p. 19). Utilizando la definición de la Comisión Europea (2006, p. 15), asumimos que:

"La competencia digital entraña el uso seguro y crítico de las tecnologías de la sociedad de la información (TSI) para el trabajo, el ocio y la comunicación. Se sustenta en las competencias básicas en materia de TSI: el uso de ordenadores para obtener, evaluar, almacenar, producir, presentar e intercambiar información, y comunicarse y participar en redes de colaboración a través de Internet".

De modo similar la LOU (RD 1512/2006) incorpora a la legislación española sobre el sistema educativo la importancia de la Competencia Digital entendida como las habilidades para transformar la información en conocimiento.

Posteriormente la LOMCE (RD 126/2014) introduce siete competencias clave y entre ellas la digital. Además, reconoce de modo explícito la importancia de las TIC para introducir cambios metodológicos y redundar en la mejora de la calidad de la enseñanza.

A partir de su propio trabajo inicial de definición de la Competencia Digital, la Comisión Europea ha continuado explorando este constructo y ha elaborado un marco conceptual para su análisis conocido como "Marco para el Desarrollo y el Competencia digital: una necesidad del profesorado universitario en el siglo XXI. M ${ }^{a}$ Paz Prendes Espinosa, Isabel Gutiérrez Porlán y Francisco Martínez Sánchez. Página 10 de 22 
Conocimiento de la Competencia Digital en Europa" (2013), marco que fue posteriormente revisado en 2016 y que ha sido utilizado por el INTEF para elaborar su propio modelo de Competencia Digital Docente (véase la Figura 4).

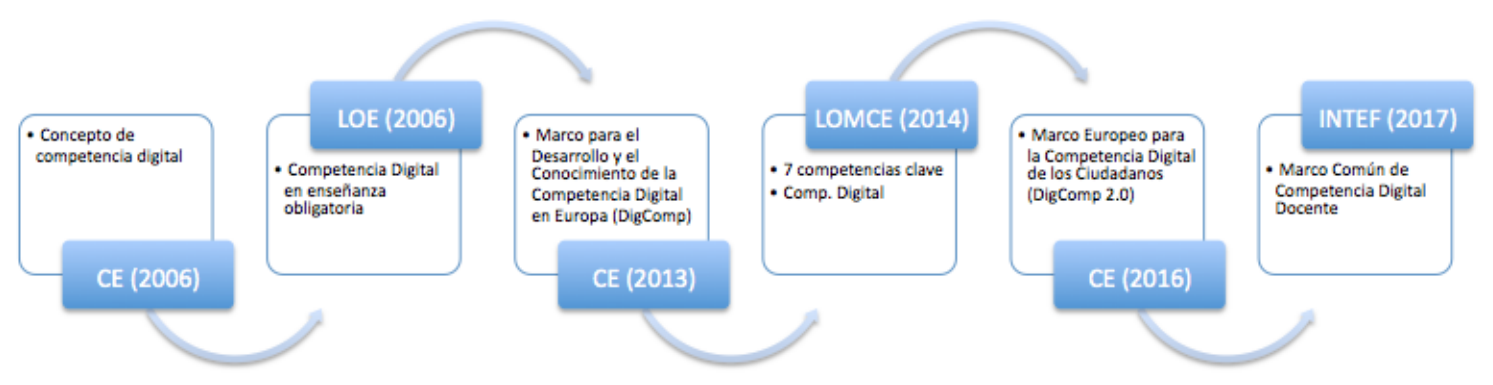

Figura 4: Marco normativo europeo y español sobre Competencia Digital.

Ese modelo de competencia digital ha sido adaptado para construir el modelo DigCompOrg de las Organizaciones Digitalmente Competentes (Comisión Europea, 2016), en el cual aparecen siete áreas clave y 15 subelementos que a su vez incluyen 74 descriptores, muchos de ellos centrados en las acciones de los agentes educativos, pero también otros relativos infraestructuras y recursos, a modelos de dirección, a roles, a estructuras organizativas y a planes de gobernanza. Este concepto de organización digitalmente competente recuerda al concepto de la "organización que aprende" y la "organización basada en competencias" del trabajo de Tejada y Navío (2005), apareciendo así el concepto de "competencia colectiva" frente a la competencia individual que usamos de modo habitual y que, en el planteamiento de los autores, tiene incluso más relevancia que ésta última. Estas ideas conducen a Tejada y Navío a pergeñar un modelo organizativo de gestión por competencias.

De modo general y a partir de un análisis exhaustivo de definiciones de competencia digital (Gutiérrez, 2014), podemos concluir que las definiciones de competencia TIC o competencia digital pueden clasificarse en relación a dos grandes perspectivas: por un lado, las que enfatizan el componente tecnológico y, por otro lado, las que hacen hincapié en la dimensión informacional o comunicativa. Y finalmente podemos acordar que la competencia digital puede ser entendida como "valores, creencias, conocimientos, capacidades y actitudes para utilizar adecuadamente las tecnologías, incluyendo tanto los ordenadores como los diferentes programas e Internet, que permiten y posibilitan la búsqueda, el acceso, la organización y la utilización de la información con el fin de construir conocimiento" (Gutiérrez, 2014, p. 54).

En lo que respecta a los docentes, hablamos de "competencia digital docente", como una competencia que se inscribe dentro del marco de las competencias profesionales del profesorado. En palabras de Tejada (2009, p. 12), es importante considerar las TIC en nuestra reflexión sobre el papel del docente porque con ellas "queda trastocado, por no decir radicalmente transformado" su rol profesional. Además el autor añade que, en la situación actual, "se tendrá que abogar por el dominio de nuevas competencias profesionales que garanticen tanto el saber, como el saber hacer, el saber estar y el saber hacer en y con TIC". 
En el contexto de la docencia, por tanto, se entiende que un docente en la actualidad debe poseer competencias para el uso adecuado de las tecnologías como profesional de la enseñanza que es. En definición de Krumsvik (2011, p. 44),

"la competencia digital es la competencia del profesor/formador de profesores en el uso de las TIC en un contexto profesional con buen criterio pedagógico-didáctico y su conciencia de sus implicaciones para las estrategias de aprendizaje y la formación digital de los alumnos y estudiantes"

Otra definición interesante y también desde una perspectiva globalizadora es la de Carrera y Coiduras $(2012$, p. 15) para quienes la competencia digital docente incluye:

"conocimientos, capacidades, actitudes y estrategias que [...] el profesor o el educador debe ser capaz de activar, adoptar y gestionar en situaciones reales para facilitar el aprendizaje alcanzando mayores niveles de logro y promover procesos de mejora e innovación permanente en los procesos formativos".

La competencia TIC o competencia digital referida a los docentes va mucho más allá del conocimiento sobre cómo usar las tecnologías, pues supone conocimientos y capacidades para poder llevar a cabo procesos de selección e integración curricular de estas tecnologías.

Es interesante observar que, en la legislación española, en la Ley Orgánica de Educación (RD 2/2006, Artículo 102) se hace referencia explícita a las tecnologías cuando se alude a la formación permanente del profesorado, indicando que "Las Administraciones educativas promoverán la utilización de las tecnologías de la información y la comunicación". Y posteriormente (en el Artículo 111bis) se alude de nuevo a las Tecnologías de la Información y la Comunicación indicando que los entornos virtuales de aprendizaje han de servir para aplicar los planes educativos y para "contribuir a la extensión del concepto de aula en el tiempo y en el espacio", además de que se deben tener en cuenta "los principios de accesibilidad universal y diseño para todas las personas". Y por último, el punto 6 del citado artículo, menciona de modo explícito que el Ministerio debe elaborar un "marco común de referencia de competencia digital docente que oriente la formación permanente del profesorado y facilite el desarrollo de una cultura digital en el aula".

Hemos llevado a cabo una revisión de numerosos autores que han trabajado la competencia digital docente (Salinas, 1998; Olcott y Schmidt, 2002; Hanna, 2002; Fernández, 2001; Badia, 2004; Mishra y Koehler, 2006, 2008; Oliveira, Cervera y Martí, 2009; Pozos, 2009; Gallego, Gámiz y Gutiérrez, 2010; Oliveira, 2010; Pozo, 2010; Prendes, 2010; Gisbert y Esteve, 2011; Gutiérrez, 2011; Gutiérrez, 2011; Krumsvick, 2011; Prendes y Gutiérrez, 2013; Esteve, 2015; Durán, Gutiérrez y Prendes, 2016a y 2016b; Gisbert, 2017; Marín, 2017). No todos ellos se centran en el profesorado universitario, pero todas sus propuestas son útiles, dado que los análisis sobre las competencias profesionales docentes presentan numerosas similitudes independientemente de los diferentes niveles de la enseñanza hacia los cuales se focalizan.

Las propuestas que de modo concreto ponen el foco en el docente universitario aparecen recogidas en la Tabla 2 y han sido analizadas en anteriores trabajos (Durán, Competencia digital: una necesidad del profesorado universitario en el siglo XXI. M ${ }^{a}$ Paz Prendes Espinosa, Isabel Gutiérrez Porlán y Francisco Martínez Sánchez. Página 12 de 22 
Gutiérrez y Prendes, 2016a y 2016b) como fruto de un análisis sistemático riguroso y exhaustivo. Tras este análisis y tomando como punto de partida nuestra propia propuesta hecha con carácter específico para el profesorado universitario no hace muchos años (véase Prendes, 2010; Prendes y Gutiérrez, 2013), hemos rediseñado el modelo de competencia digital docente del profesor universitario, el cual además de incluirlo en la última fila de la Tabla (como Prendes, 2017), lo hemos representado en la Figura 5.

Tabla 2: Competencia Digital Docente del Profesorado Universitario.

\begin{tabular}{|c|c|c|}
\hline Modelo & Autor & $\begin{array}{l}\text { Descripción Competencia TIC } \\
\text { (áreas/dimensiones/ámbitos) }\end{array}$ \\
\hline $\begin{array}{l}\text { Competencia } \\
\text { Digital del } \\
\text { Profesorado } \\
\text { Universitario } \\
\text { en su } \\
\text { Desarrollo } \\
\text { Profesional } \\
\text { Docente }\end{array}$ & $\begin{array}{l}\text { Pozos } \\
(2009)\end{array}$ & $\begin{array}{l}\text { Áreas: Planificación y diseño de experiencias de aprendizaje en } \\
\text { ambientes presenciales y virtuales; Desarrollo y conducción de } \\
\text { experiencias de aprendizaje colaborativas presenciales y en red; } \\
\text { Orientación, guía y evaluación procesos de construcción del } \\
\text { conocimiento en entornos presenciales y virtuales; Gestión del } \\
\text { crecimiento y desarrollo profesional con apoyo de las TIC; } \\
\text { Investigación, desarrollo e innovación pedagógica con/para el uso de las } \\
\text { TIC en educación; Diversidad, ética y uso responsable de las TIC en } \\
\text { desempeño profesional docente; Medio ambiente, salud y seguridad } \\
\text { laboral con el uso de las TIC en la profesión docente. } \\
\text { Fases: Básicas; Profundización; Generación del conocimiento }\end{array}$ \\
\hline $\begin{array}{l}\text { Competencia } \\
\text { s TIC para la } \\
\text { docencia en } \\
\text { la } \\
\text { Universidad } \\
\text { Pública } \\
\text { Española }\end{array}$ & $\begin{array}{l}\text { Prendes } \\
(2010)\end{array}$ & $\begin{array}{l}\text { Áreas: Conocimiento general del ordenador y posibilidades de las TIC; } \\
\text { Conocimiento y uso de las estrategias metodológicas para el trabajo en } \\
\text { red; Posibilidades y limitaciones de las TIC en el proceso de enseñanza- } \\
\text { aprendizaje; Elección recursos TIC para el aula; Conocimiento y uso de } \\
\text { herramientas; Publicación de material en la red; Uso de las TIC para } \\
\text { diferentes tareas docentes; Formación docente e innovación con TIC } \\
\text { Niveles: 1. dominio de las bases que fundamentan la acción con TIC; } 2 \text {. } \\
\text { diseño, implementación y evaluación de acción con TIC; } 3 \text {. análisis y } \\
\text { reflexión personal y colectiva de la acción llevada a cabo con TIC. }\end{array}$ \\
\hline $\begin{array}{l}\text { Competencia } \\
\text { digital } \\
\text { Universidad } \\
\text { de Lérida }\end{array}$ & $\begin{array}{l}\text { Carrera y } \\
\text { Coiduras } \\
(2012)\end{array}$ & $\begin{array}{l}\text { Conocimiento de dispositivos, herramientas informáticas y aplicaciones } \\
\text { red, y capacidad para evaluar su potencial didáctico; Diseño actividades } \\
\text { y evaluación con TIC con los estudiantes y con su contexto; } \\
\text { Implementación y uso ético, legal y responsable de las TIC; } \\
\text { Transformación y mejora de práctica profesional docente, Tratamiento y } \\
\text { gestión eficiente de la información de la red; uso de la red para trabajo } \\
\text { colaborativo y comunicación e interacción interpersonal; Ayuda } \\
\text { proporcionada a los alumnos para que se apropien de las TIC y se } \\
\text { muestren competentes en su uso }\end{array}$ \\
\hline $\begin{array}{l}\text { CDES, } \\
\text { Cuestionario } \\
\text { de Comp. } \\
\text { digitales en } \\
\text { Educación } \\
\text { Superior }\end{array}$ & $\begin{array}{l}\text { Mengual, } \\
\text { Roig y } \\
\text { Blasco } \\
(2016)\end{array}$ & $\begin{array}{l}\text { Áreas: alfabetización tecnológica; uso y acceso a la información; } \\
\text { comunicación y colaboración; ciudadanía digital; creatividad e } \\
\text { innovación. }\end{array}$ \\
\hline $\begin{array}{l}\text { Competencia } \\
\text { digital } \\
\text { docente del } \\
\text { profesor } \\
\text { universitario }\end{array}$ & $\begin{array}{l}\text { Prendes } \\
(2017)\end{array}$ & $\begin{array}{l}\text { Áreas: técnica; información y comunicación; educativa; analítica y } \\
\text { reflexiva; y, por último, social y ética. } \\
\text { Ámbitos: docencia, investigación y gestión. }\end{array}$ \\
\hline
\end{tabular}

En esta evolución de nuestro modelo hasta llegar al más reciente (Prendes, 2017) hemos mantenido los tres ámbitos de aplicación (docencia, investigación y gestión), pues todos ellos son característicos del rol profesional del PDI en las universidades 
(Personal Docente e Investigador). El cambio se produce en las dimensiones, pues ahora proponemos cinco dimensiones en lugar de las tres que aparecían en el modelo de 2010. Las cinco dimensiones de la competencia digital docente del profesor universitario son: técnica; informacional y comunicativa; educativa; analítica; y por último, social y ética (véase la Figura 5).

Aunque a priori las dimensiones no representan un nivel específico de dificultad, sí se podría interpretar que en la práctica pudiera haber una relación. En otras palabras, el proceso de desarrollo se podría poner en relación con los niveles progresivos de perfeccionamiento y capacitación:

1. La competencia técnica puede ser un primer nivel de competencia que, una vez adquirida, nos permitirá la capacitación para el dominio de las tecnologías en relación con la información y la comunicación.

2. En una fase posterior, seremos capaces de aprovechar esta competencia para incorporar las tecnologías en el contexto educativo (tanto para diseñar y gestionar ambientes mediados por tecnologías como para promover aprendizajes enriquecidos con TIC o desarrollar procesos de interacción, colaboración y evaluación con estos medios).

3. Y ya en niveles más avanzados del desarrollo de la competencia digital, seremos capaces de reflexionar de modo crítico sobre nuestra propia competencia y gestionar nuestros procesos de formación permanente y de desarrollo profesional.

4. También en un nivel avanzado seremos conscientes del impacto social y cultural de las tecnologías, así como de dimensiones éticas y de seguridad que con bastante probabilidad en los primeros niveles de la competencia sean más difíciles de desarrollar.

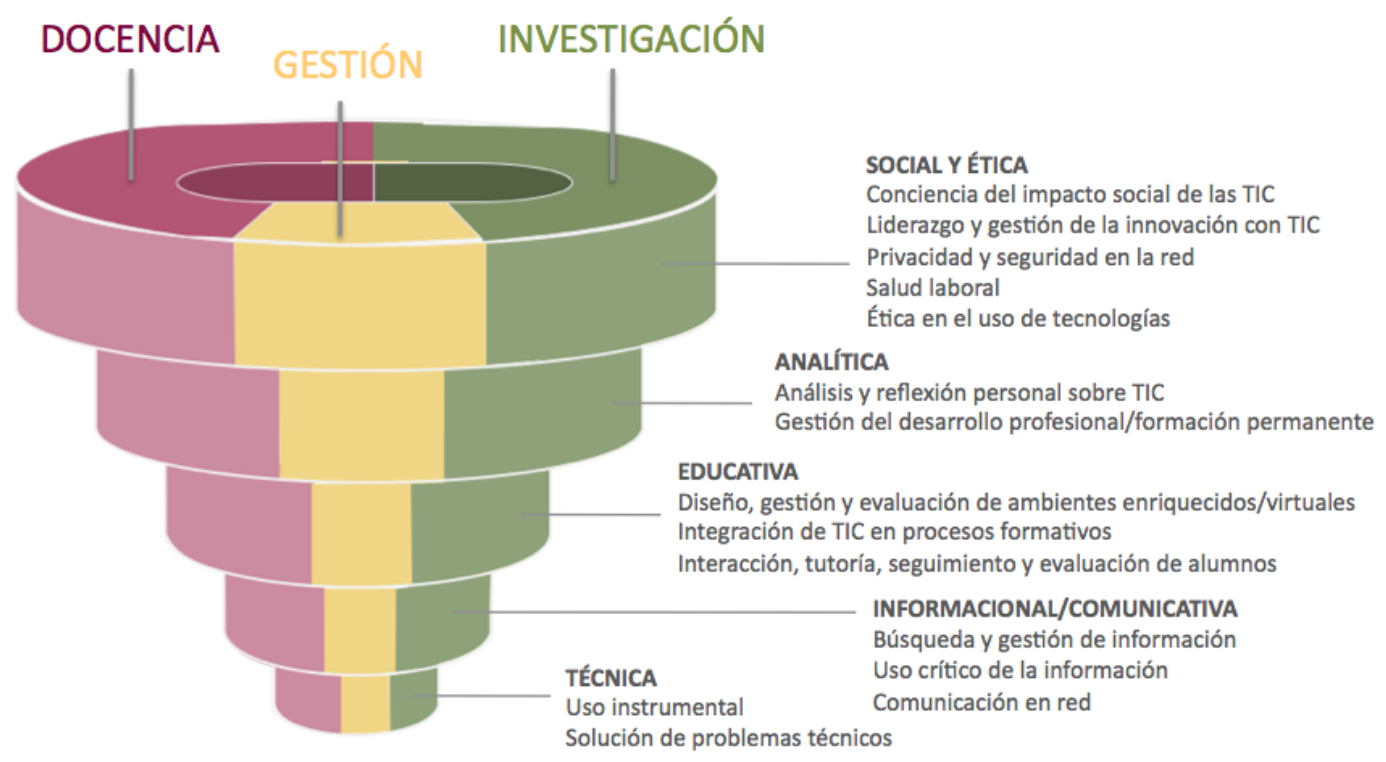

Figura 5: Modelo de Competencia Digital Docente del Profesor Universitario (tomado de Prendes, 2017, p. 87).

La relación entre las cinco dimensiones y los diversos niveles (de principiante a experto) requeriría de una investigación para su comprobación empírica. No obstante, consideramos que puede ser un modelo de interés tanto para los trabajos teóricos como 
para trabajos de carácter empírico, pues es el resultado de la revisión sistemática de modelos anteriores y de un análisis de contenido de sus diversas propuestas.

Nuestro modelo no describe niveles de dominio de la competencia y en este sentido nos parece una propuesta muy interesante la de Carretero, Vuorikari y Punie (2017), quienes toman como base el modelo DigComp que ya hemos mencionado y concretan ocho niveles de dominio usando para ello como sustento la conocida taxonomía de Bloom.

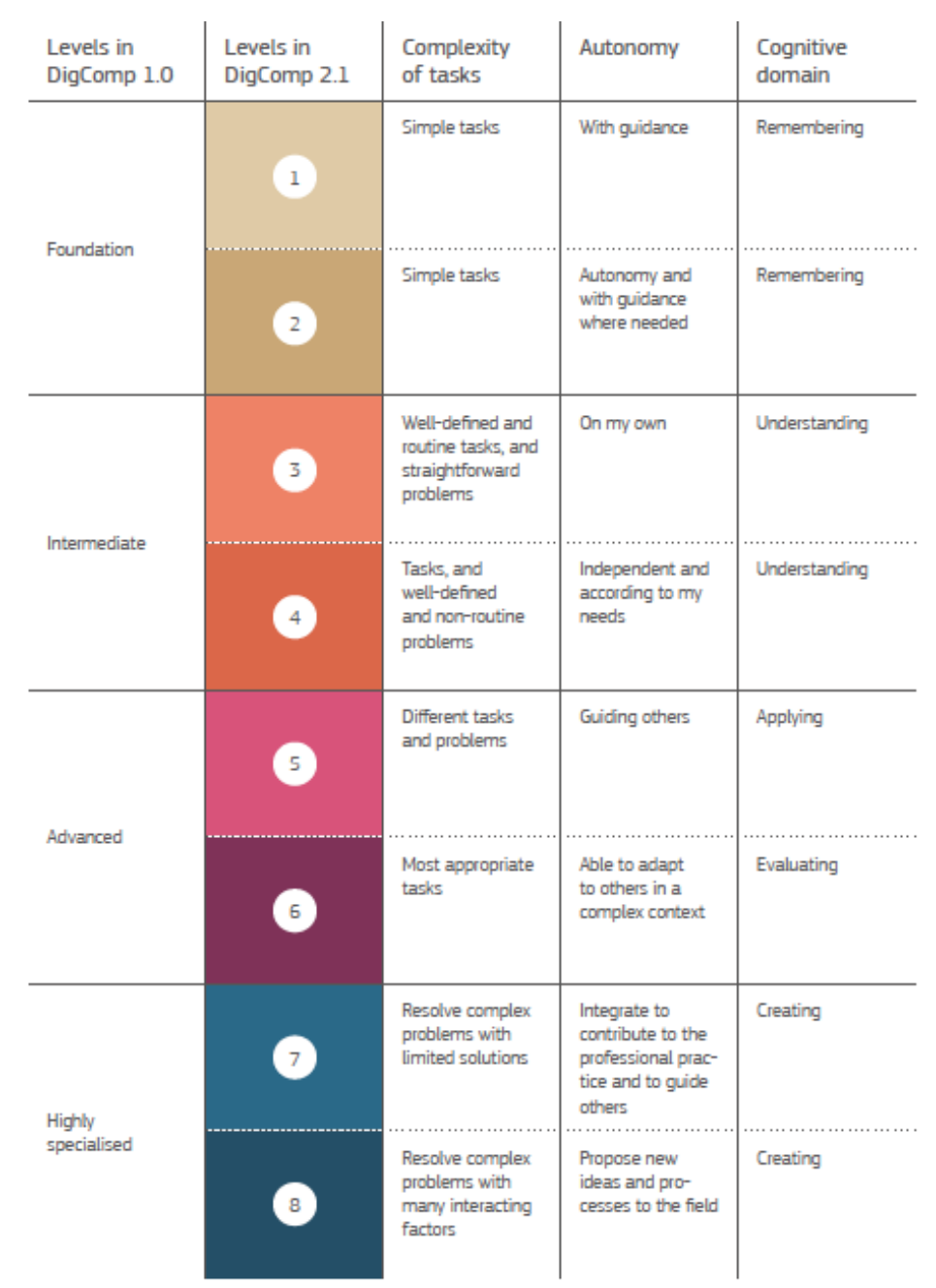

Figura 6: Niveles de dominio de la Competencia Digital (tomado de Carretero, Vuorikari y Punie, 2017, p. 13).

\section{A modo de conclusión}

Las universidades que quieran realmente introducir innovaciones significativas en el ámbito de la formación apoyada en TIC y que quieran tener una plantilla de profesorado innovador y con competencias digitales, deberán apostar seriamente por una redefinición de sus planes estratégicos y aplicar medidas que redunden de forma eficaz en el logro de sus objetivos. Medidas que redefinan el trabajo actual del profesor universitario, pues el mucho tiempo empleado en la burocracia y en la gestión administrativa -mal entendida como calidad- es tiempo que no podemos emplear en lo 
que de verdad es nuestra misión: la formación y la investigación. En otras palabras, medidas para que cada zapatero pueda dedicarse a sus zapatos.

Hay una cuestión general que afecta directamente a la formación docente del profesor universitario y, casi con total seguridad, podemos afirmar que provoca en él una inquietud. En la medida en que la acción docente no sea valorada por la administración y la propia institución de igual manera que la actividad investigadora, difícilmente se podrá lograr que se demande y alcance la capacitación que parece necesaria en el momento actual. Eso supone que se estará metiendo vino de reserva en odres viejos. Lo cual es una forma de estropearlo.

El difícil equilibrio entre la docencia y la investigación es un elemento característico del profesorado universitario y debe ser contemplado y convenientemente valorado por nuestras instituciones. Es bien cierto que se han dado algunos pasos en este sentido, pero no suficientes.

Además de lo anterior y en línea con el asunto central de nuestro artículo, se hace necesario apostar por una formación del profesorado que asuma la relevancia de la competencia digital y que tome como punto de partida una adecuada comprensión de lo que supone ser un docente competente digitalmente. La formación centrada en la parte más técnica -una consecuencia de la apuesta de las universidades por la dotación tecnológica-, hace que olvidemos la dimensión realmente importante relativa a cómo usar, analizar y reflexionar sobre esas tecnologías para utilizarlas de forma efectiva en los procesos de enseñanza-aprendizaje, en la gestión y en la investigación.

Si retomamos el modelo de competencia digital propuesto en este trabajo, observamos cómo las acciones formativas casi siempre se quedan en el primero de los niveles (capacitación técnica); a veces trabajan en el segundo nivel del uso de herramientas para la gestión de la información y la comunicación; menos veces trabajan la capacitación en el uso educativo y las metodologías docentes; y casi nunca llegan a los niveles analítico y ético. Deberemos por tanto redefinir las competencias profesionales del docente universitario, integrando en ellas la competencia digital, y deberemos hacerlo desde un enfoque holístico que contemple todas las dimensiones y ámbitos de la misma.

Por otra parte, se requieren medidas estructurales y organizativas que sirvan para reconstruir una maquinaria oxidada y obsoleta. Es necesario comprender los requerimientos organizativos de las nuevas universidades, instituciones donde se pueda flexibilizar la oferta formativa y donde podamos, por fin, construir comunidades de aprendizaje más abiertas al exterior: a la sociedad, al mundo de la empresa, al mundo del conocimiento y al mundo en el cual nuestros alumnos serán protagonistas de su desarrollo en breve plazo cuando sean egresados.

Y por último, medidas para que el alumnado pueda desarrollar sus capacidades y competencias como ciudadano del siglo XXI. Dejemos de ser compartimentos estancos y facilitemos las experiencias de aprendizaje en entornos flexibles enriquecidos con tecnologías. 
En relación con la formación del alumnado, queremos hacer una llamada de atención sobre el diseño de nuestros planes de estudio, donde a menudo confluyen intereses personales y de grupos de poder más que los intereses de los propios alumnos. Es triste comprobar que no hemos aprendido de los errores del pasado y seguimos tropezando, una y otra vez, en las mismas piedras. Es probable que en estos procesos de diseño curricular en la universidad nos encontremos en ocasiones con quienes destaquen "por su clarividencia y altura de miras", pero también es probable que nos encontremos muy a menudo con aquellos otros que destacarán "por su codicia y miopía" (Trillo, 2008, p. 23).

Y si esperamos que la ANECA reconduzca esta tendencia de una mal entendida autonomía universitaria, creemos que no vamos a poder ir por el camino correcto. Deben ser las propias universidades quienes establezcan mecanismos de control que garanticen la mejor formación para nuestros alumnos y garanticen la adquisición de las competencias que prometemos sobre el papel, entre ellas la competencia digital que aparece como competencia genérica en todas las universidades españolas... pero no siempre la vamos a encontrar reflejada en nuestros planes de estudios, tristemente.

De comprender el papel que la Universidad ha de jugar en su entorno social depende la propia supervivencia de las universidades. E indefectiblemente, las tecnologías están llamadas a ser grandes protagonistas de ese papel de las universidades del siglo XXI.

Presentación del artículo: 20 de diciembre de 2017 Fecha de aprobación: 17 de enero de 2018 Fecha de publicación: 31 de enero de 2018

Prendes Espinosa, M.P., Martínez Sánchez, F. y Gutiérrez Porlán, I. (2017). Competencia digital: una necesidad del profesorado universitario en el siglo XXI. RED Revista de Educación a Distancia, 56. Consultado el (dd/mm/aaaa) en http://www.um.es/ead/red/56/prendes_et_al.pdf

\section{Financiación}

Esta investigación no ha recibido ninguna subvención específica de los organismos de financiación en los sectores públicos, comerciales o sin fines de lucro.

\section{Referencias bibliográficas}

Amador Muñoz, L. V.; Cárdenas-Rodríguez, R. y Terrón-Caro, T. (2017). Introducción: Innovación docente en el ámbito de la Universidad. Revista de Humanidades, 31, 11-15. Recuperado de DOI: 10.5944/rdh.31.2017.19070

Aramburuzabala Higuera, P., Martínez Garrido, C. y García Peinado, R. (2013). La formación del profesorado universitario en España: evolución y perspectiva. Educación, 22(43), 7-25. 
Badia, A. (2004). Las competencias docentes en el uso formativo de las TIC. En Badia, A. Del docente presencial al docente virtual. Barcelona: UOC.

Cabero, J. y Barroso, J. (2016). Formación del profesorado en TIC. Una visión del modelo TPACK. Cultura y Educación, 28(3), 647-663. DOI: 10.1080/11356405.2016.1203526

Cabero, J., Marín, V. y Castaño, C.M. (2015). Validación de la aplicación del modelo TPACK para la formación del profesorado en TIC. @TIC, revista d'innovació educativa, (14), 13-22. DOI: 10.7203/attic.14.4001

Carrera, F.X. y Coiduras, J.L. (2012). Identificación de la competencia digital del profesor universitario: un estudio exploratorio en el ámbito de las ciencias sociales. REDU. Revista de Docencia Universitaria, 10(2), 273-298. Recuperado de: http://red-u.net/redu/index.php/REDU/article/view/383

Carretero, S., Vuorikari, R. y Punie, Y. (2017). DigComp 2.1. The digital Competence Framework for Citizens. With eight proficiency levels and examples of use. Recuperado de Comisión Europea website http://publications.jrc.ec.europa.eu/repository/bitstream/JRC106281/webdigcomp2.1pdf_(online).pdf

Cebrián de la Serna, M. (2013). La innovación educativa vs. la innovación tecnológica. En A. Villa Rodriguez (Eds.), Las Universidades como generadoras de la Innovación: Investigación, iniciativa y responsabilidad social (pp. 499516). Foro Internacional sobre Innovación Universitaria.

Comisión Europea (2006). Competencias clave para el aprendizaje permanente. Recomendación 2006/962/CE del Parlamento Europeo y del Consejo, de 18 de diciembre de 2006, sobre las competencias clave para el aprendizaje permanente [Diario Oficial L 394 de 30.12.2006]

Comisión Europea (2014). Comprender las políticas de la Unión Europea: Agenda Digital para Europa. Recuperado de http://europa.eu/pol/index_es.htm

Comisión Europea (2016). DigCompOrg. Digitally Competent Educational Organisations. Recuperado de https://ec.europa.eu/jrc/en/digcomporg

Durán, M., Gutiérrez, I. y Prendes, M.P. (2016a). Análisis conceptual de modelos de competencia digital del profesorado universitario. RELATEC, Revista Latinoamericana de Tecnología Educativa, 15(1), 97-114.

Durán, M.; Gutiérrez, I. y Prendes, M.P. (2016b). Certificación de la Competencia TIC del Profesorado Universitario: Diseño y validación de un instrumento. Revista Mexicana de Investigación Educativa, 21(6), 527-556

Escudero, J.M. (2006). La formación del profesorado y la garantía del derecho a una buena educación para todos. En J.M. Escudero y A. Luis (Coords.), La formación del profesorado y la mejora de la educación para todos: políticas y prácticas (pp. 21-51). Barcelona: Octaedro. 
Esteve, F. (2015). La competencia digital docente: análisis y evaluación del desempeño de los estudiantes universitarios de educación por medio de un entorno $3 D$ (Tesis Doctoral). Universidad Rovira i Virgili, Tarragona. Recuperado de: http://francescesteve.es/tesis/

Eurydice (2011). Key Data on Learning and Innovation through ICT at School in Europe 2011. Recuperado de http://eacea.ec.europa.eu/education/eurydice

Flores-Lueg, C. B. y Roig-Vila, R. (2016). Competencia digital docente: una cuestión clave para la educación del siglo XXI. En J. Gómez-Galán, E. López-Meneses y L. Molina-García (Eds.), Instructional Strategies in Teacher Training (pp. 8798). UMET Press. Recuperado de https://www.researchgate.net/publication/288003681_Instructional_Strategies_i n_Teacher_Training

Fernández, M.C. (2001). Los medios audiovisuales, informáticos y nuevas tecnologías en los centros educativos gallegos: presencia y usos, Santiago de Compostela, Facultad de Ciencias de la Educación, tesis doctoral inédita.

Gallego, M.J., Gámiz, V. y Gutiérrez, E. (2010). El futuro docente ante las competencias en el uso de las tecnologías de la información y comunicación para enseñar. EDUTEC, Revista de Tecnología Educativa, 34, 1-18. DOI: 10.21556/edutec.2010.34.418

Gisbert, M. (2017). Una Tecnología Educativa para la Era Digital. Manuscrito no publicado, Proyecto de Cátedra. Universidad Rovira i Virgili, Tarragona.

Gisbert, M. y Esteve, F. (2011). Digital learners: La competencia digital de los estudiantes universitarios. La Cuestión Universitaria, 7, 48-59

Gómez, J. (2016). UNIVERSITIC 2016. Análisis de las TIC en las Universidades Españolas. Madrid: CRUE Universidades Españolas. Recuperado de http://tic.crue.org/wp-content/uploads/2017/04/UNIVERSITIC-2016-conportadas.pdf

Gutiérrez Porlán, I. (2011). Competencias del profesorado universitario en relación al uso de tecnologías de la información y comunicación: Análisis de la situación en España y propuesta de un modelo de formación (Tesis Doctoral). Universidad Rovira i Virgili, Tarragona. Recuperado de http://hdl.handle.net/10803/52835

Gutiérrez Porlán, I. (2014). Perfil del profesor universitario español en torno a las competencias en tecnologías de la información y la comunicación. Pixel-Bit, Revista de Medios y Educación, 44, 51-65. DOI: 10.12795/pixelbit.2014.i44.04

Hanna, D. (2002). Nuevas perspectivas sobre el aprendizaje en la enseñanza universitaria. En Hanna, D. La enseñanza universitaria en la era digital. (pp. 5983). Barcelona: Octaedro.

ITE, Instituto de Tecnologías Educativas (2010). Habilidades y competencias del siglo XXI para los aprendices del nuevo milenio en los países de la OCDE (EDU Working paper no. 41). Recuperado de 
http://recursostic.educacion.es/blogs/europa/media/blogs/europa/informes/Ha bilidades_y_competencias_siglo21_OCDE.pdf

Krumsvik, R. (2011). Digital competence in Norwegian teacher education and schools. Högre utbildning, 1(1), 39-51. Recuperado de http://journals.lub.lu.se/index.php/hus/article/view/4578

Ley Orgánica de Educación (LOE) (Ley Orgánica 2/2006, 3 de mayo). Boletín Oficial del Estado, $n^{\circ}$ 106, 2006, 4 mayo

Marcovitch, J. (2002). La universidad (im)posible. Cambridge: Cambridge University Press

Marín Trejo, R. (2017). Diseño y evaluación de un instrumento de la competencia digital docente (Tesis Doctoral). Universidad de Islas Baleares.

Marín, I., Román, P., Barroso, J. y Castaño, C. (2002). Necesidades formativas del profesorado universitario para la utilización de las tecnologías de la formación y la comunicación. Revista de Psicodidáctica, (14), 161-172. Recuperado de http://www.ehu.eus/ojs/index.php/psicodidactica/article/view/152/148

Mas, O. (2011). El profesor universitario: sus competencias y formación. Profesorado, revista de currículum y formación del profesorado, 15(3), 195-211. Recuperado de http: //www.ugr.es/local/recfpro/rev153COL1.pdf

Mishra, P. y Koehler, M. (2006). Technological pedagogical content knowledge: A Framework for Teacher knowledge. Teachers College Record, 108(6), 10171054. http://punya.educ.msu.edu/publications/journal_articles/mishra-koehlertcr2006.pdf

NMC - New Media Consortium (2017). NMC Horizon Report: 2017 Higher Education Edition. Recuperado de http://cdn.nmc.org/media/2017-nmc-horizonreport-he-EN.pdf

OCDE, Organización para la Cooperación y el Desarrollo Económico, (2005). The definition and selection of key competences. Executive Summary. Recuperado de http://www.oecd.org/pisa/35070367.pdf. Traducción al español en http://deseco.ch/bfs/deseco/en/index/03/02.parsys.78532.downloadList.94248.D ownloadFile.tmp/2005.dscexecutivesummary.sp.pdf

Olcott, Jr. y Schmidt, K. (2002). La redefinición de políticas y prácticas del profesorado en la era del conocimiento. En Hanna, D. La enseñanza universitaria en la era digital (pp. 265-291). Barcelona: Octaedro.

Oliveira, J. (2010) Pre-service teacher education enriched by technology-supported learning environments: a learning technology by design approach. Journal of Literacy and Technology, 11,1.

Oliveira, J., Cervera, M., Martí, M. (2009). Learning as representation and representation as learning: A theoretical framework for teacher knowledge in the digital age. Proceedings of World Conference on Educational Multimedia, 
Hypermedia and Telecommunitcations 2009, pp 2646-2653. Chesapeake, VA: $\mathrm{AACE}$

Pagés, T., Hernández, C., Abadía, A.R., Bueno, C., Ubieto-Artur, I., Márquez, D., Sabaté, S. y Jorba, H. (2016). La innovación como competencia docente en la Universidad: innovación orientada a la mejora de aprendizaje. Revista de Psicología, Ciencies de l'Edució i de l'Esport, 34(1), 33-43.

Perrenoud, P. (2004). Diez nuevas competencias para enseñar. Barcelona: Graó.

Pozos, K. (2010). La Competencia Digital del Profesorado Universitario para la Sociedad del Conocimiento: Aproximación a un Modelo y Validación de un Cuestionario de Detección de Necesidades de Formación Continua. Manuscrito no publicado, Doctorado en Calidad y Procesos de Innovación Educativa, Depto. Pedagogía Aplicada, Universidad Autónoma de Barcelona

Prendes Espinosa, M.P. (2010). Competencias TIC para la docencia en la Universidad pública española: Indicadores y propuestas para la definición de buenas prácticas. Informe del proyecto financiado por la Secretaría de Estado de Universidades e Investigación del Ministerio de Educación dentro de la convocatoria Estudio y Análisis (EA2009-0133). Recuperado de http://www.um.es/competenciastic

Prendes Espinosa, M.P. y Gutiérrez Porlán, I. (2013). Competencias tecnológicas del profesorado en las universidades españolas. Revista de Educación, 361, 196-222. Recuperado de http://dide.minedu.gob.pe/xmlui/handle/123456789/2432

Prendes Espinosa, M.P. (2017). La competencia digital: nuevos actores en los nuevos espacios de formación. Una mirada desde la Tecnología Educativa. Proyecto de Cátedra (inédito). Universidad de Murcia.

Ricoy, M.C., Sevillano, M.L. y Feliz, T. (2008). Competencias necesarias para la utilización de las principales herramientas de Internet en educación. Revista de Educación, 356, 483-507. Recuperado de http://www.revistaeducacion.educacion.es/re356/re356_20.pdf

Salinas, J. (1998). El rol del profesor universitario ante los cambios de la era digital. Agenda Académica, 5(1), 131-141.

Salinas, J. (2004). Innovación docente y uso de las TIC en enseñanza universitaria. RUSC, Revista de Universidad y Sociedad del Conocimiento, 1(1), 1-16. Recuperado de https://www.uoc.edu/rusc/dt/esp/salinas1104.pdf

Santos Rego, M. A., Jover Olmeda, G., Naval, C., Álvarez Castillo, J.L., Vázquez Verdera, V. y Sotelino Losada, A. (2017). Diseño y validación de un cuestionario sobre práctica docente y actitud del profesorado universitario hacia la innovación (CUPAIN). Educación XXI, 20(2), 39-71. DOI: 10.5944/educXX1.17806

Tejada, J. (2009). Competencias docentes. Profesorado. Revista de currrículum y formación del profesorado, 13(2), 1-15. Recuperado de 
https://www.ugr.es/ recfpro/rev132COL2.pdf

Tejada, J. y Navío, A. (2005). El desarrollo y la gestión de competencias profesionales: una mirada desde la formación. Revista Iberoamericana de Educación, 37(2), 1-15. Recuperado de http://www.rieoei.org/deloslectores/1089Tejada.pdf

Todorov, T. (2008). El miedo a los bárbaros. Barcelona: Galaxia Gutenberg.

Trillo, F. (2008). El reto de las titulaciones de educación. Otra crónica a 7 de marzo del 2008. Educación XXI, 11, 19-41. Recuperado de http://revistas.uned.es/index.php/educacionXX1/article/view/308/264

UNESCO (2004). Las Tecnologías de la Información y la Comunicación en la formación docente. Guía de Planificación. Recuperado de http://unesdoc.unesco.org/images/0012/001295/129533s.pdf

UNESCO (2016). Educación para la Ciudadanía Mundial. Preparar a los educandos para los retos del siglo XXI. París. Recuperado de http://unesdoc.unesco.org/images/0024/002449/244957s.pdf

Villa Sánchez, A. (2008). La excelencia docente. Revista de Educación, número extraordinario, 177-212.

Zabalza, M. A. (2003). Competencias docentes del profesorado universitario. Madrid: Narcea.

Zabalza, M.A. (2007). Competencia docente del profesor universitario: calidad y desarrollo profesional. Madrid: Narcea. 